\title{
L'immersion fictionnelle au-delà de la narrativité
}

\section{Gabrielle Trépanier-Jobin et Alexane Couturier}

\section{(2) OpenEdition \\ Journals}

Édition électronique

URL : http://journals.openedition.org/sdj/950

DOI : $10.4000 /$ sdj. 950

ISSN : 2269-2657

Éditeur

Laboratoire EXPERICE - Centre de Recherche Interuniversitaire Expérience Ressources Culturelles Education

\section{Référence électronique}

Gabrielle Trépanier-Jobin et Alexane Couturier, «L'immersion fictionnelle au-delà de la narrativité », Sciences du jeu [En ligne], 9 | 2018, mis en ligne le 01 juin 2018, consulté le 30 avril 2019. URL : http:// journals.openedition.org/sdj/950; DOI : 10.4000/sdj.950

Ce document a été généré automatiquement le 30 avril 2019.

Tous droits réservés 


\title{
L'immersion fictionnelle au-delà de la narrativité
}

\author{
Gabrielle Trépanier-Jobin et Alexane Couturier
}

1 Dans l'industrie du jeu vidéo, l'immersion du joueur est l'un des effets les plus recherchés par les développeurs et un argument de vente de taille que les marketeurs n'hésitent pas à invoquer. Lorsque le mot «immersion » est employé pour faire la promotion des jeux, c'est plus souvent en référence à leur graphisme « kinoréaliste » et à la complexité de leur intelligence artificielle qu'à leur trame narrative engageante (Therrien, 2013). Depuis l'arrivée récente, sur le marché, des dispositifs de réalité virtuelle avec vision stéréoscopique à 360 degrés, l'immersion dans les jeux vidéo est plus que jamais associée à l'illusion de réalisme et à la saturation des sens (Therrien, 2017). Il ne faudrait cependant pas oublier que le sentiment d'immersion dans un jeu vidéo ne repose pas uniquement sur un appareillage technologique sophistiqué. Comme c'est le cas des lecteurs de romans et des spectateurs de films, les joueurs peuvent également s'immerger dans l'univers fictionnel d'un jeu.

2 Plusieurs chercheurs se sont déjà intéressés au rôle que jouent la dimension narrative et les personnages dans la construction d'une expérience vidéoludique immersive (Murray, 1997 ; Ryan, 2001; Calleja, 2011; Therrien, 2011; Arsenault, 2013 ; Georges, 2013; Therrien, 2013). Force est toutefois de constater que l'immersion fictionnelle dans les jeux vidéo est souvent étudiée en marge de l'immersion sensorielle et de l'immersion basée sur le défi. Pour bien comprendre la complexité du phénomène de l'immersion, il semble pourtant nécessaire de prendre en considération la possible coexistence de l'immersion fictionnelle avec ces autres formes d'immersion lors d'une expérience vidéoludique. Dans cet article, nous aimerions démontrer que l'immersion fictionnelle ne peut être étudiée qu'en fonction de la diégèse, du récit et des personnages. En prenant comme point de départ le modèle systémique de l'immersion développé par Calleja (2011), de même que le SCI-model d'Ermi et Mäyrä (2005), nous défendrons l'idée qu'il faut tenir compte de la manière dont le jeu crée un équilibre entre ses différentes dimensions (narrative, ludique, spatiale, kinesthésique) afin de favoriser l'engagement narratif, l'identification au personnage et, ultimement, l'immersion fictionnelle. Pour illustrer la pertinence d'une 
théorie sur l'immersion fictionnelle qui tienne compte de l'interaction complexe entre différentes dimensions d'un jeu, nous effectuerons une analyse détaillée du jeu d'aventure et d'exploration sous-marine à visée immersive Abzû (Giant Squid, 2016) en nous basant sur une approche ludonarrative.

\section{Définitions et théories de l'immersion}

\section{Un terme polysémique}

3 Comme l'explique Janet Murray dans Hamlet on the Holodeck, l'utilisation du mot « immersion » pour décrire le sentiment d'être transporté dans un environnement simulé repose sur un emploi métaphorique du terme "immersion" (du latin immersio), qui signifie littéralement être submergé dans un liquide (Murray, 1997, p. 98). L'auteure compare ce sentiment à celui qu'on peut éprouver lorsqu'on plonge dans l'océan et qu'on se retrouve soudainement entouré d'une réalité complètement différente. Abordée dans de nombreux champs d'études tels que la littérature, le cinéma et la psychologie, l'immersion revêt, au fil du temps et des technologies disponibles à une époque donnée, diverses significations qui en enrichissent le sens, mais en complexifient la définition et la compréhension de ses effets. Il existe d'ailleurs un important flou conceptuel et de nombreux débats terminologiques autour de cette notion polysémique.

Bien que les termes "immersion" et "présence " réfèrent globalement au même phénomène expérientiel, ils n'ont pas la même signification d'un champ disciplinaire à l'autre. En cyberpsychologie, le terme "immersion" réfère à la capacité d'une technologie à créer une forte illusion de réalité, alors que le terme " présence » renvoie à l'effet psychologique de cette technologie et au sentiment de transport dans un univers autre qui en découle lorsque l'usager arrive à oublier momentanément où il se trouve (Slater et Wilbur, 1997, p. 606). Cherchant à éviter le déterminisme technologique, les chercheurs en sciences humaines et sociales ont, pour leur part, tendance à utiliser le terme immersion pour référer à l'état psychologique qui consiste à se sentir « enveloppés et inclus dans un environnement avec lequel on interagit et qui procure un flot continu de stimulus et d'expériences " (Witmer et Singer, 1998, p. 227, notre traduction).

5 En études du jeu vidéo, la plupart des chercheurs envisagent l'immersion comme un état psychologique, mais la signification exacte du terme demeure imprécise, puisqu'il est utilisé pour référer à deux sentiments distincts. Ceux qui parlent d'immersion dans un jeu comme Tetris (Pajitnov, 1984), où le joueur doit contrôler des objets sans que sa présence dans l'environnement du jeu ne soit reconnue et où l'espace vidéoludique est entièrement visible à l'écran, la considèrent comme un sentiment d'« absorption dans une condition, action, intérêt, etc.» (Calleja, 2011, p. 26, notre traduction). Ceux qui discutent d'immersion dans un jeu comme Tomb Raider (Eidos Interactive, 1996), où l'espace est navigable et où le joueur est représenté, la conçoivent plutôt comme un sentiment de transport dans un univers autre (Calleja, 2011, p. 28, notre traduction). Alors que l'immersion comme transport peut être vue comme un synonyme de ce que les chercheurs en cyberpsychologie appellent "présence ", on ne peut pas en dire autant pour le sentiment d'absorption, que certains chercheurs, comme Marie-Laure Ryan (2001, p. 9), n'incluent pas dans leur conception de l'immersion. L'immersion fictionnelle est généralement associée au sentiment de "présence " dans un univers diégétique, mais nous verrons qu'elle peut aussi, dans le cas des jeux vidéo, être étroitement liée au 
sentiment d'absorption lorsque l'histoire du jeu se présente comme un puzzle à reconstituer.

\section{Le modèle holistique de Calleja}

6 Cherchant à surmonter cette confusion autour du terme "immersion », Gordon Calleja propose d'utiliser le terme moins fortement connoté « incorporation » pour qualifier un état psychologique intense, mais fragile et intermittent, lors duquel la conscience du joueur et l'environnement du jeu ne font plus qu'un. Avec ce terme, Calleja entend référer à un phénomène double : l'assimilation de l'environnement du jeu dans l'esprit du joueur et l'incorporation [embodiment] du joueur dans l'univers du jeu, concourant tous deux à l'impression d'habiter les lieux. Étant donné la description qu'il fait du concept, l'incorporation ne s'applique qu'aux médias interactifs qui reconnaissent la présence du joueur dans l'environnement du jeu et lui confère une certaine agentivité (Calleja, 2011, pp.169-173). Calleja avance aussi l'idée qu'un engagement simultané avec plusieurs dimensions du jeu est nécessaire à l'incorporation et que l'engagement avec chacune de ces dimensions implique que le joueur n'y porte plus consciemment attention (pp. 40-41). Lorsqu'un joueur commence une partie, son attention est souvent focalisée sur sa maitrise des contrôles et sa familiarisation avec l'espace, ce qui rend l'incorporation difficile durant cette phase d'apprentissage (pp. 170-171).

7 Parmi les différents types d'engagements relevés par Calleja, on retrouve : l'engagement kinesthésique, qui concerne la maîtrise et l'intériorisation des contrôles; l'engagement spatial, lié à la structure des lieux et à la familiarisation du joueur avec la géographie du jeu ; l'engagement narratif, qui concerne l'intérêt pour le récit scénarisé et l'histoire générée par les actions du joueur ; l'engagement affectif, référant aux émotions suscitées par le jeu; l'engagement ludique, concernant les buts poursuivis par le joueur, ses possibilités d'actions, ses choix et les conséquences qui en découlent; et enfin, l'engagement partagé, regroupant l'interaction, la coopération et la compétition avec d'autres joueurs - qui sera mis de côté dans le présent article puisque nous analysons un jeu solo (Calleja, 2011, pp. 43-44). Cette subdivision est utile pour étudier le phénomène de l'immersion, car elle permet de mieux comprendre comment les différentes dimensions d'un jeu (interface physique, spatialité, narrativité, jouabilité) peuvent susciter cet état psychologique. Il ne faudrait toutefois pas croire que ces dimensions agissent chacune indépendamment sur l'immersion du joueur. Comme l'indique Calleja, elles «sont expérimentées non pas isolément, mais toujours en relation les unes aux autres, la séparation étant ici fait à des fins d'analyse " (Calleja, 2011, p. 37, notre traduction). L'auteur reconnaît donc que les différentes composantes d'un jeu produisent des effets combinés sur l'immersion, même s'il étudie l'impact de chacune d'elles séparément dans son livre (chaque chapitre porte sur une composante en particulier).

8 Le modèle de Calleja servira de point de départ à notre réflexion parce qu'il prend à la fois en considération l'expérience subjective du joueur et les aspects formels du jeu, en plus d'admettre que plusieurs variables interagissent pour susciter l'incorporation. Contrairement à Calleja, nous préférons conserver le terme «immersion » pour nous référer tantôt au sentiment d'absorption, tantôt au sentiment de présence du joueur, selon le type d'immersion qui est expérimenté. Puisque Calleja étudie des jeux de rôle en ligne massivement multijoueur (MMORPGs) qui ont tendance à favoriser un engagement simultané avec toutes les dimensions d'un jeu, son modèle rend 
moins bien compte des jeux qui développent davantage certaines dimensions et misent sur un type d'immersion en particulier. Il exclut par ailleurs la possibilité même d'une « immersion fictionnelle » puisqu'il relègue le sentiment d'être transporté par l'histoire d'un jeu au statut d'« engagement narratif». Pour toutes ces raisons, nous nous appuierons également sur le "SCI-model » développé par Laura Ermi et Frans Mäyrä (2005).

\section{Le « SCI-Model » d'Ermi et Mäyrä}

9 Dans leur texte «Fundamental Components of the Gameplay Experience: Analysing Immersion ", Ermi et Mäyrä (2005) identifient trois types d'immersion possibles lors d'une expérience vidéoludique. L'immersion sensorielle repose sur le degré de vivacité des stimuli audiovisuels qui surpassent en intensité l'information sensorielle provenant du monde physique (Ermi et Mäyrä, 2005, p.7). Elle est favorisée par la saturation perceptuelle, l'illusion de réalisme audiovisuel, la transparence du médium et l'isolation du joueur des stimuli provenant de son entourage (Therrien, 2014, p. 451). L'immersion imaginative - qu'Arsenault et Picard (2008) qualifient d'immersion fictionnelle - implique que le joueur se sente transporté dans la diégèse du jeu par le biais de l'histoire, des personnages et des représentations (Ermi et Mäyrä, 2005, p. 8). Elle repose sur la plausibilité de l'univers fictionnel, la crédibilité des personnages, la familiarité du spectateur avec les conventions employées, la cohérence des événements et le déploiement de stratégies visant à renforcer l'effet de tension, de choc, de tristesse, etc. (Therrien, 2014, p. 454). Ces deux types d'immersion ne sont pas exclusifs au jeu vidéo et peuvent survenir lors du visionnement d'un film ou d'une capsule de réalité virtuelle non interactive. Partant de l'idée que les règles et la jouabilité sont au cœur de l'expérience vidéoludique, Ermi et Mäyrä (2005, p.7) ajoutent à ces deux sous-catégories de l'immersion une troisième qui est spécifique au jeu vidéo : l'immersion basée sur le défi. De manière similaire au concept de flow théorisé par le psychologue Csikszentmihalyi (1990), celle-ci prend forme lorsqu'un équilibre se crée entre les habiletés du joueur et le degré de difficulté des défis présents dans le jeu. Ce type d'immersion - qu'Arsenault et Picard (2008) appelle l'immersion systémique - repose, d'une part, sur la clarté des instructions et des buts à atteindre, sur la rétroaction du système et sur la nature engageante des mécaniques de jeu et se fonde, d'autre part, sur les habitudes vidéoludiques du joueur, sa compréhension des règles, sa dextérité et sa capacité à résoudre les défis cognitifs ou physiques proposés par le jeu (Therrien, 2014, pp. 452-453). Généralement, l'immersion sensorielle et l'immersion fictionnelle sont liées au sentiment d'être transporté dans un univers autre, alors que l'immersion basée sur le défi est associée au sentiment d'absorption dans une certaine condition se produisant lorsque nos ressources mentales sont tellement sollicitées par une tâche qu'on oublie, pour un instant, où l'on se trouve. Nous verrons toutefois que ces deux sentiments peuvent se confondre lorsque le joueur doit fournir un effort mental pour reconstituer l'histoire du jeu.

10 Le modèle d'Ermi et Mäyrä nous semble particulièrement intéressant parce qu'il prend à la fois en considération les composantes du jeu, les particularités du joueur et le contexte de jeu, mais aussi parce qu'il admet la possibilité d'un jeu qui privilégie l'aspect fictionnel ou encore la résolution de problèmes pour susciter l'immersion. Sa tripartition peut donner l'impression que les trois types d'immersion sont mutuellement exclusifs, mais 
comme les auteurs le mentionnent au passage, ils peuvent se mélanger et s'enchevêtrer de différentes façons (Ermi et Mäyrä, 2005, p. 8). Ermi et Mäyrä (2005, p. 11) expliquent que, dans certains jeux et genres vidéoludiques, une forme d'immersion prend le dessus sur les autres en raison du développement accru de certaines dimensions; ce qui a d'ailleurs été confirmé par une étude d'Arsenault et Picard (2008). Dans le prolongement de leur modèle, nous tenterons de mieux comprendre comment l'immersion fictionnelle interagit avec les autres types d'immersion et comment les composantes de jeu autres que la narrativité et les personnages influencent l'immersion fictionnelle. Avant d'y arriver, il est toutefois nécessaire de résumer comment l'immersion fictionnelle est considérée dans les études du jeu vidéo.

\section{L'immersion fictionnelle dans les jeux vidéo}

11 L'immersion fictionnelle dont il est ici question est entendue dans un sens différent de celui que lui confère Jean-Marie Schaeffer. Dans son ouvrage Qu'est-ce que la fiction?, le théoricien décrit l'immersion fictionnelle comme un état mental «biplanaire» lors duquel le joueur se laisse volontairement tromper par les illusions pour entrer dans la fiction, tout en maintenant une saine distance critique pour éviter d'acquérir de fausses croyances (Schaeffer, 1999, p. 190). Autrement dit, l'immersion fictionnelle consiste à se laisser prendre au jeu, tout en sachant qu'il ne s'agit que d'un jeu. Même si nous sommes d'accord avec Schaeffer pour dire que l'immersion fictionnelle implique de se laisser leurrer sans perdre tout jugement critique, nous ne concevons pas l'immersion fictionnelle comme porte d'entrée vers la fiction ni comme condition de son existence. L'immersion fictionnelle dont nous faisons mention fait référence à une forme d'expérience vidéoludique possible lorsque les conditions optimales sont réunies, autant du côté du jeu que du joueur.

\section{Trois sous-catégories d'immersion fictionnelle dans les jeux vidéo}

12 À la suite de Marie-Laure Ryan (2001), Arsenault et Picard (2008) subdivisent l'immersion fictionnelle dans les jeux vidéo en trois sous-catégories. Reliée à l'exploration spatiale, l'immersion diégétique - que Ryan nomme l'immersion spatiale - renvoie à l'impression d'être présent dans l'univers fictionnel d'un jeu et serait influencée par la qualité et la quantité des détails fournis dans cet espace (Arsenault et Picard, 2008, p. 12). La familiarité du joueur avec l'environnement du jeu contribuerait également à la naissance de ce sentiment. L'immersion narrative - que Ryan appelle l'immersion temporelle réfère au sentiment d'être captivé par le récit et d'être impatient de connaître son dénouement. Elle serait liée aux stratégies narratives mises en place dans le jeu et au désir qu'a le joueur de faire progresser l'histoire (Ibid.). L'immersion identificatrice - que Ryan appelle l'immersion émotionnelle - surviendrait, pour sa part, lorsque le joueur a l'impression d'incarner son personnage ou lorsqu'il établit un lien d'attachement fort avec lui suscitant des émotions intenses et complexes (Arsenault et Picard, 2008, p. 13). Parmi les dimensions du jeu qui sont généralement associées à l'immersion fictionnelle, on retrouve la narrativité et les personnages. Ces deux dimensions comportent différentes variables censées influencer positivement ou négativement l'immersion fictionnelle. 


\section{Influence de la narrativité sur l'immersion fictionnelle}

13 Le terme narrativité englobe traditionnellement les notions d'histoire, de récit et de narration, qui ne sont pas utilisées comme des synonymes :

l'histoire est une série d'évènements qui touchent un ou plusieurs personnages et qui se déroulent dans un monde donné, le récit est l'organisation de cette histoire en un texte et le style dans lequel il est produit, et la narration, quant à elle, est le mode de communication du récit. (Arsenault, 2006, p. 19)

Ces distinctions n'ont toutefois pas la même pertinence pour le médium vidéoludique, dont l'histoire se déroule la plupart du temps ici et maintenant. Tel que le remarque Ryan (2006), les productions vidéoludiques ne sont pas des histoires au sens classique du terme, mais constituent des systèmes capables de générer du contenu narratif. Puisque le joueur a le double rôle de spectateur qui regarde l'histoire se dérouler sous ses yeux et d'acteur qui influence le déroulement de l'histoire, jouer une partie revient à actualiser « l'une des possibles histoires que permet le système» (2006, p. 281, notre traduction). Même lorsqu'un jeu comporte une seule trame narrative possible, le joueur doit performer des actions pour enclencher son déroulement. Comme l'explique Arsenault, le mode narratif d'un jeu se déploie toujours en fonction d'un algorithme qui est non seulement responsable de l'application des règles, mais aussi » de structurer l'enchaînement des contenus et d'informer le joueur sur ce qu'il advient du monde fictionnel et des actions qu'il entreprend» (Arsenault, 2006, p. 97). Dans bien des cas, le jeu vidéo est " extrinsèquement" narratif, au sens où il peut véhiculer un récit au moyen de techniques cinématographiques ou littéraires, et « intrinsèquement » narratif, au sens où les actions du joueur ont une portée narrative au sein de l'univers diégétique (pp. 97-98).

Pour rendre compte de ce phénomène, Calleja (2011, pp.114-115) distingue l'histoire scénarisée du jeu (scripted narrative) de l'alterbiographie du joueur. L'histoire scénarisée est celle qui a été préalablement intégrée dans le code de programmation par les concepteurs du jeu et peut être véhiculée de deux façons distinctes. Dans un type de récit que Levine (2008) appelle le "push narrative », les informations sur l'histoire peuvent être imposées aux joueurs par le biais de cinématiques, d'actions contextuelles (quick time events), de textes ou de dialogues. Dans un type de récit que Levine (2008) nomme le «pull narrative ", le joueur doit plutôt découvrir des informations dissimulées et disséminées dans l'univers du jeu pour les réorganiser en un tout cohérent et émettre des hypothèses sur l'histoire (cité dans Calleja, 2011, pp.122-123). Même si, note Calleja, le récit à reconstituer implique le risque que certains joueurs passent à côté d'une partie du contenu narratif, ceux ayant pris le temps et fourni l'effort de chercher les éléments narratifs seront davantage investis dans l'histoire.

16 L'alterbiographie (aussi appelée narrativité expérientielle ou récit en devenir) est, quant à elle, l'histoire générée par les actions du joueur et continuellement actualisée dans son esprit de façon plus ou moins consciente (Calleja, 2011, pp. 115-117). Selon Jenkins (2004, p. 128), ces «récits émergents » (emergent narratives), qui prennent forme au fur et à mesure que le joueur entreprend des actions dans le monde fictionnel, ne sont pas prédéterminés par les concepteurs, mais demeurent limités par le design du jeu. L'alterbiographie est donc non seulement influencée par la capacité d'imagination du joueur, mais aussi par la marge de liberté qui lui est octroyée et par le degré d'ouverture $\mathrm{du}$ monde vidéoludique. Pour reprendre la terminologie de Chandler (2007), le développement d'une riche alterbiographie serait favorisé par une structure narrative 
«mythocentrique » ouverte dans laquelle le joueur peut poursuivre ses propres objectifs, plutôt que par une structure "logocentrique " linéaire et planifiée d'avance par les concepteurs (Chandler cité in Arsenault, 2014, p. 480). Au lieu d'envisager ces deux structures narratives comme des catégories binaires, indique Arsenault (2014), il faut les concevoir comme un continuum: une structure narrative n'est jamais entièrement fermée ou ouverte, mais se situe quelque part entre ces deux extrêmes d'une manière qui peut fluctuer au cours de la progression du joueur. Selon Calleja (2011, pp.131-133), l'engagement narratif a plus de chance de se produire lorsque l'alterbiographie du joueur se marie bien avec les composantes de l'histoire scénarisée, lorsque cette dernière tient le joueur en haleine et lorsqu'elle l'intéresse suffisamment pour qu'il inclue ses éléments dans son alterbiographie.

\section{Influence du personnage-joueur sur l'immersion fictionnelle}

Comme le souligne Arsenault (2006, p. 6), c'est à travers le personnage-joueur que nous pouvons découvrir l'histoire d'un jeu, s'y immerger et participer à l'élaboration d'un récit interactif rendant l'expérience vidéoludique plus engageante. Tout comme les concepts d'immersion et de narrativité, les termes " personnage » et " avatar » sont polysémiques (Aldred, 2014, p. 356). Certains chercheurs appellent "avatar» la "représentation visuelle de la présence du joueur dans l'univers du jeu» et «acteur» le "personnage distinct du joueur, avec sa personnalité, ses caractéristiques et, jusqu'à un certain point, sa propre pensée » (Gard cité in Arsenault, 2013, p. 255). Dans les études du jeu vidéo, il est courant d'associer l'avatar aux jeux à la première personne dans lesquels le joueur vit lui-même les événements et l'acteur, aux jeux à la troisième personne dans lesquels le joueur se distingue de son personnage tout en le contrôlant (Arsenault, 2013). Comme le démontrent Arsenault (2013) et Therrien (2013), le point de vue (première ou troisième personne) n'est toutefois pas le seul facteur influençant la relation entre le joueur et son personnage, étant donné le déploiement d'une pluralité d'autres stratégies par les concepteurs. Les rapports entre un joueur et un personnage de jeu vidéo sont beaucoup plus complexes et se situent généralement quelque part sur un continuum entre deux postures distinctes, soit l'extension de soi et l'identification.

La posture de l'«extension de soi» (Therrien, 2013, p. 227) suppose que le joueur se projette dans le monde fictionnel du jeu par le biais d'un avatar qu'il perçoit comme un prolongement numérique de son corps. Pour expliquer cette relation entre le joueur et son avatar, Jessica Aldred (2014, p. 357) utilise la notion d'incarnation (embodiment) et explique que le joueur considère être le personnage (identify as), plutôt que de s'identifier à lui comme s'il s'agissait d'une entité séparée (identify with). L'extension de soi correspond à ce que Jean-Marie Schaeffer nomme la "virtualisation identitaire »; une posture selon laquelle «le sujet fictionnel qui se déplace dans l'univers virtuel est en fait un double du joueur réel » (Schaeffer cité in Arsenault, 2013, p. 4). Selon Therrien (2013), cette posture est favorisée par des avatars «coquille vide» qui ont des traits caractéristiques peu développés (ou personnalisables) de même que des possibilités d'action étendues. Dans le même ordre d'idée, Arsenault (2013) associe cette posture aux « jeux à dominante avatorielle » qui minimisent l'altérité entre le personnage et le joueur. Selon Salen et Zimmerman (2004, pp. 453-455), le joueur qui se met à la place de son personnage demeure néanmoins conscient qu'il s'agit d'une entité fictive pouvant être 
contrôlée à sa guise telle une marionnette. C'est ce que les auteurs appellent la « double conscience du joueur ».

19 La posture de l'«identification» (Therrien, 2013, p. 227) suppose, quant à elle, que le joueur éprouve un lien d'attachement ou de l'empathie envers un personnage, tout en le considérant comme une entité distincte de lui. L'identification correspond à ce que Schaeffer appelle l'« allosubjectivité »; une posture selon laquelle le joueur " s'identifie à un personnage fictif qui se déplace dans le monde fictionnel en accord avec les ordres que le joueur lui donne » (cité par Arsenault, 2013, p.4). Selon Therrien, cette posture est favorisée par des « avatars caractérisés » dont la personnalité est prédéterminée par les concepteurs du jeu et dont les expressions ou réactions sont perceptibles à l'écran. Voir le visage expressif de l'avatar ou constater les dommages faits à son enveloppe corporelle a tendance à déclencher la peur, la tension ou l'empathie réflexe chez le joueur doté d'un système neuronal miroir favorisant la «contagion émotionnelle» (Grodal cité in Therrien, 2013, pp. 236-237). Puisque la majorité du temps, le joueur voit son avatar de dos, ce sont souvent les personnages non joueurs qui déclenchent ces émotions (Therrien, 2013, p. 238). Dans le même ordre d'idée, Arsenault (2013) associe cette posture aux jeux à dominante actorielle qui préservent les différences intersubjectives entre le personnage et le joueur. Bien évidemment, chaque joueur réagit différemment et peut osciller entre ces deux postures au cours de sa partie.

Ce qui complique également la donne, c'est que des stratégies compensatoires qu'Arsenault (2013, p. 258) appelle des "marqueurs de subjectivation» - sont parfois utilisées pour renforcer la fusion identitaire dans les jeux à dominante actorielle. Parmi ces stratégies, on retrouve le protagoniste silencieux, le protagoniste amnésique qui découvre son histoire en même temps que le joueur, le récit de naissance ou d'enfance du personnage, la caméra qui fait voir ce que le personnage voit, le jet de sang qui éclabousse l'objectif, les boîtes de texte qui reflètent les pensées du personnage, etc. À l'inverse, des stratégies compensatoires - qu'Arsenault (2013, pp. 256-257) appelle des «marqueurs d'allosubjectivité » - sont parfois employées pour préserver l'altérité entre le joueur et le personnage dans les jeux à dominante avatorielle. Parmi ces stratégies, on peut compter le son de la voix du personnage, les passages fréquents de la caméra en troisième personne, les cinématiques, les reflets du visage de l'avatar sur des vitres ou des miroirs, etc.

21 Selon Therrien (2013), la présence vidéoludique est favorisée par un avatar caractérisé mis en scène selon des stratégies narratives qui, contrairement aux cinématiques, intègrent des possibilités d'action. Autrement dit, l'immersion fictionnelle dans un jeu vidéo a plus de chance de se produire lorsque le personnage-joueur a ses "préoccupations propres qui ne font pas simplement écho à la volonté de puissance qui anime le joueur » (Therrien, 2013, p. 245). De manière similaire, Arsenault (2013, p. 270) affirme que l'approche actorielle demeure la «clé de voûte » de l'immersion fictionnelle et le meilleur «allié » du joueur dans son expérience ludonarrative vidéoludique, même s'il restreint sa liberté de dire et de faire ce qu'il lui plaît. Pour susciter l'immersion fictionnelle dans un jeu vidéo, il peut néanmoins être profitable de créer un équilibre entre les approches actorielle et avatorielle, indique Arsenault. La liberté d'agir du joueur et sa capacité à exprimer ses préférences de jeu seront comblées par l'approche avatorielle, alors que l'approche actorielle renforcera la force émotive du récit. Pour donner lieu à un bon récit interactif et favoriser l'immersion fictionnelle, il serait donc 
préférable de "jouer sur les deux fronts », c'est-à-dire avoir " une mesure d'acteur dans l'avatar et une mesure d'avatar dans l'acteur » (Arsenault, 2013, p. 258).

\section{Influence d'autres dimensions du jeu sur l'immersion fictionnelle}

22 Au début des années 2000, il existait un important débat entre les narratologues, qui croyaient en la possibilité d'utiliser les concepts issus de la narratologie pour étudier les récits vidéoludiques (Murray, 1997), et les ludologues, selon lesquels il fallait inventer de nouveaux outils conceptuels pour étudier les récits interactifs des jeux vidéo (Aarseth, 1997 ; Frasca, 1999 ; Juul, 2001). Ce débat a eu un grand impact sur le développement subséquent des études du jeu vidéo et sur la manière d'aborder le concept de narrativité (Ryan, 2006, p. 275). Aujourd'hui, la plupart des théoriciens du jeu s'entendent pour dire que le monde fictionnel motive le joueur à atteindre les buts du jeu et que l'interactivité génère le récit plutôt que d'exclure sa possibilité d'existence. Ils reconnaissent, toutefois, que les jeux sont plus souvent centrés autour de la jouabilité qu'autour de l'histoire ; que le système de règles encadre les éléments fictionnels et leur confère un sens; que le récit a une fonction différente dans les jeux et les médias traditionnels; et que certains genres vidéoludiques sont axés sur la jouabilité alors que d'autres sont focalisés sur la narrativité (Calleja, 2011, p. 13 ; Arsenault, 2014, p. 476).

Depuis que le débat entre les ludologues et les narratologues est révolu, plusieurs auteurs s'efforcent de faire le pont entre les dimensions ludique et narrative d'un jeu vidéo. Dans Avatars of Story, Ryan (2006, p.291) propose d'adopter une approche ludonarrative consistant à : concevoir l'histoire comme un moyen de faciliter l'apprentissage des règles; se demander si le système de règles pourrait être lié à une histoire différente ; chercher à voir si les règles sont cohérentes avec le monde fictionnel, etc. Dans Half-Real, Juul (2005, p. 149) adopte également une approche ludonarrative pour étudier comment le système de règles confère un sens aux éléments fictionnels en donnant, entre autres, l'exemple du jeu Call of Duty IV (Infinity Ward, 2007) dans lequel le drapeau n'a pas la signification traditionnelle d'identité nationale et de patriotisme, mais plutôt celle de la victoire. Juul indique, par ailleurs, que les concepts d'espace vidéoludique, de design et de quête font le pont entre les règles et la fiction (Juul, 2005, p. 17 et 188). On pourrait en dire autant du concept d'alterbiographie (ou de récit émergent), qui rend bien compte du fait que les actions du joueur sur le plan ludique contribuent à l'élaboration de l'histoire. Arsenault (2013, p. 262) fait remarquer que le récit et l'avatar sont à la base de la jouabilité, car ils justifient et délimitent les possibilités d'actions du joueur de même que le répertoire de stratégies qu'il peut mettre à exécution, en plus de motiver ses actions. À la lumière de l'approche ludonarrative, il ne semble pas suffisant, pour étudier l'immersion fictionnelle, de concentrer uniquement son attention sur la structure narrative du jeu, sur ses cinématiques, sur le type d'avatar privilégié et sur le lien que le joueur est encouragé à tisser avec son personnage.

Quelques auteurs ont déjà fait le pont entre l'immersion fictionnelle et les dimensions du jeu autres que la narrativité et les personnages. Le concept d'immersion diégétique, développé par Arsenault et Picard (2008, p. 12) à la suite de Ryan (2001), par exemple, prend en considération l'influence de la spatialité sur l'immersion fictionnelle : plus un environnement vidéoludique est riche en couleurs, en textures et en stimuli audiovisuels, plus il sera facile pour le joueur de croire au monde fictionnel. Ce concept laisse entrevoir la possible coexistence de l'immersion sensorielle et diégétique, dans la mesure où elles 
sont toutes deux favorisées par un environnement riche en détail. Dans sa thèse de doctorat, Therrien (2011, p. 79) avance l'idée que l'association des boutons de la manette à différents membres du corps de l'avatar (main, jambe, cou), tel que c'est le cas dans le jeu Heavy Rain (Quantic Dream, 2010), aide le joueur à percevoir l'avatar comme une extension de son corps. Ce faisant, il met en évidence le rôle que peut jouer l'interface physique et les contrôles dans l'immersion identificatoire. En mentionnant les tracées en surbrillance, les flèches directionnelles et les cartes spationarratives qui indiquent au joueur dans quelle direction aller pour faire avancer sa mission et, par le fait même, l'histoire du jeu, Therrien (2011, p. 74 et 84) laisse entrevoir le rôle que peut jouer l'interface graphique dans l'immersion narrative. Lorsqu'il mentionne les jeux qui diégétisent la formation du joueur en intégrant les instructions aux répliques d'un personnage non joueur, par exemple, Therrien met également en évidence la possibilité d'entrer en immersion fictionnelle dès la phase d'apprentissage du jeu (p. 80). À la suite de Schaeffer (1999), selon qui l'immersion fictionnelle requiert une "attitude ludique " impliquant de "faire comme si », Genvo $(2006$, p.6) défend l'idée que la jouabiltié ( gameplay), soit l'actualisation des règles du jeu par le joueur, encourage cette attitude ludique. On pourrait aussi penser que la jouabilité influence l'immersion narrative lorsque les récompenses prennent la forme d'éléments narratifs comme: une cinématique, une rencontre avec un nouveau personnage ou l'accès à une nouvelle intrigue.

À notre avis, de nombreux autres éléments non narratifs peuvent influencer l'immersion fictionnelle dans un jeu vidéo. L'analyse du jeu solo Abzû nous permettra de mieux saisir l'impact de la dimension ludique, spatiale et kinesthésique sur la capacité du joueur à concentrer son attention sur l'histoire et sur les personnages pour mieux s'immerger dans l'univers fictionnel. Nous avons entre autres choisi ce jeu parce que sa visée immersive est clairement énoncée dans son paratexte. Ainsi peut-on lire dans la description du jeu sur la plateforme Steam : «Immergez-vous dans un monde débordant de couleur et de vie dans votre descente vers le cœur de l'océan ».

\section{Analyse d'Abzû}

Notre analyse ludonarrative du jeu Abzû s'inscrit dans une démarche exploratoire visant à proposer des pistes de réflexion sur les facteurs pouvant influencer l'immersion fictionnelle dans un jeu vidéo. Nous ne prétendons pas rendre compte de l'éventail des expériences qu'il est possible de vivre en jouant à ce jeu. Nous cherchons plutôt à évaluer son potentiel immersif en fonction de ses éléments constitutifs et de leur interinfluence, tout en sachant que ce potentiel immersif peut être actualisé ou non par les joueurs. Avant de procéder à l'analyse, nous avons constitué une grille regroupant différentes dimensions d'un jeu (narrativité, personnage, jouabilité, spatialité et contrôles) qui selon les théories susmentionnées - sont censées influencer l'immersion en fonction d'une série de variables. Notre travail s'inscrit également dans une démarche inductive, puisqu'il s'agit d'identifier d'autres variables pouvant influencer l'immersion fictionnelle qui ne figurent pas dans la littérature sur le sujet. Enfin, l'analyse se fonde sur nos propres expériences avec le jeu et sur nos propres interprétations de son histoire. Une étude de réception serait donc nécessaire pour voir si le potentiel immersif d'Abzû s'actualise chez la majorité des joueurs et si notre conception de son histoire fait écho à celle d'autres joueurs. 


\section{Présentation d'Abzû et de sa visée immersive} jeu d'aventure et d'exploration Abzû invite les joueurs à s'immerger, au sens propre ${ }^{1}$ comme au sens figuré, dans un monde sous-marin tridimensionnel riche en détails visuels et sonores. Présenté comme "une aventure sous-marine époustouflante qui évoque le rêve de la plongée ", Abzû laisse au joueur le soin de deviner la quête du personnage principal au fur et à mesure qu'il progresse dans le jeu. La description du jeu sur la plateforme de distribution en ligne Steam évoque l'exploration de " paysages épiques » et la découverte d'écosystèmes marins. On y retrouve également des explications sur le titre du jeu qui veut dire, en sumérien, «l'océan du savoir » et qui laisse présager un univers fictionnel riche en significations.

\section{Influence de la narrativité sur les différents types d'immersion}

La structure narrative du jeu Abzû est davantage logocentrique que mythocentrique, sans être complètement fermée et linéaire. Le joueur peut temporairement dévier de la trame narrative principale, qui consiste à sillonner la mer afin de lui rendre son énergie bleue et restituer la vie sous-marine. Il peut, en effet, explorer les recoins de l'océan, ramasser des coquillages, s'agripper au dos des tortues, faire virevolter les bancs de poissons ou méditer sur des statues. La possibilité d'errer dans le jeu et d'interagir avec les robots et les créatures sous-marines (quoique de façon limitée) permet au personnage-joueur de développer une riche alterbiographie en phase avec l'histoire scénarisée, ce qui est théoriquement censé compenser la linéarité de la trame narrative principale et renforcer l'immersion fictionnelle du joueur.

La méthode par laquelle l'histoire intégrée d'Abzû est divulguée consiste en un astucieux mélange de récit imposé (push narrative) et de récit à reconstituer (pull narrative). En effet, une bonne partie du récit doit être recomposée au moyen d'une série d'indices dispersés ici et là dans l'environnement du jeu et auxquels le joueur doit porter attention pour élaborer des hypothèses sur l'histoire. Le jeu débute avec l'image d'un mystérieux personnage inanimé qui flotte au milieu de l'océan. Après avoir pris le contrôle de ce personnage, le joueur commence à nager dans l'environnement sous-marin sans trop savoir où il se trouve et où il doit se rendre. Lors de son passage dans un temple inondé, le joueur attentif et curieux remarquera les fresques qui tapissent les murs de l'édifice et prendra le temps de les observer. Il discernera alors les représentations énigmatiques des habitants d'une civilisation disparue, qui exploitent l'énergie bleue de l'océan pour ensuite la retourner à sa source, et supposera possiblement que ces habitants vivaient en harmonie avec la nature. Dans un autre temple inondé, il apercevra l'image d'une personne qui ressemble à son avatar et qui se tient devant une structure pyramidale recouverte de circuits électroniques et au pied duquel s'agenouillent les habitants de la civilisation disparue. Faute d'indices supplémentaires, le joueur ressortira probablement du temple avec plus de questions en tête que de réponses.

Arrivé à un endroit sombre et sans vie où s'entassent des centaines de structures pyramidales qui menacent de l'électrocuter, le joueur pourra être tenté de les concevoir comme des éléments perturbateurs de l'écosystème marin. Il pensera potentiellement que ces tétraèdres électroniques épuisent les ressources d'énergie bleue permettant à cet 
environnement de prospérer et pourra prêter aux concepteurs du jeu l'intention d'illustrer un conflit entre la machine et la nature. Après être entré dans une carcasse de métal labyrinthique où se trouve une sorte d'antichambre, le joueur aura la possibilité d'activer un hologramme qui révèle la composition interne de son personnage. En voyant son squelette de métal, il comprendra peut-être que l'avatar est un androïde et verra désormais le jeu comme l'illustration d'un combat entre la machine et la machine. Les fresques, les tétraèdres explosifs et l'hologramme sont autant d'indices permettant au joueur de reconstituer l'histoire du jeu et théoriquement censés favoriser l'immersion fictionnelle chez les joueurs qui y prêtent attention.

31 Certains éléments de l'histoire sont, pour leur part, imposés au joueur par le biais de cinématiques ou d'actions contextuelles (quick time events), dont celles mettant en scène un requin qui : mange le robot-guide du personnage-joueur d'un geste menaçant puis s'enfuit, se retrouve coincé sous un tétraèdre jusqu'à ce que l'avatar vienne le secourir, se fait électrocuter en même temps que lui par un tétraèdre explosif géant et meurt sous le regard abattu de l'avatar. Ces cinématiques contribuent à l'immersion fictionnelle identificatoire en suscitant de l'empathie réflexe envers le personnage-joueur et le requin, tout comme elles favorisent l'immersion narrative en piquant la curiosité du joueur et en lui donnant envie de connaître la suite de l'histoire. Puisque la cinématique mettant en scène la mort du requin laisse entrevoir le squelette de métal du personnagejoueur à travers sa combinaison de plongée déchirée sous l'impact de l'explosion, elle fournit également des indices sur sa nature d'androïde. Cet exemple démontre qu'une cinématique énigmatique peut faire partie du récit à reconstituer plutôt que du récit imposé lorsqu'elle fournit des clefs interprétatives. Vers la fin du jeu, plusieurs cinématiques visuellement grandioses, accompagnées d'une musique enivrante, font voir la nature qui se revitalise après la destruction des tétraèdres électroniques par le personnage-joueur. Ces cinématiques concourent non seulement à l'immersion fictionnelle en servant de clefs interprétatives pour élaborer des hypothèses sur l'histoire du jeu, mais également à l'immersion basée sur le défi en récompensant le joueur et à l'immersion sensorielle en déclenchant une orgie de couleurs, de sons et de formes.

\section{Influence du personnage sur l'immersion fictionnelle}

Pour prendre part à l'aventure que propose Abzû, le joueur doit contrôler, à partir d'un point de vue à la troisième personne, un personnage humanoïde mystérieux dont le nom, le genre et le récit de vie demeurent inconnus. Affublé d'un équipement de plongée noir et jaune d'allure futuriste, le personnage ne possède aucune personnalité prédéfinie et communique de manière limitée avec les robots et les animaux qui l'entourent par le biais d'ondes sonores indéchiffrables à l'oreille humaine. L'avatar n'est pas personnalisable : ses caractéristiques physiques et ses compétences ne peuvent pas être changées par le joueur. Pour reprendre les termes proposés par Therrien (2013), le personnage se situe à mi-chemin entre l'avatar coquille vide et l'avatar caractérisé. Le fait que l'on n'ait aucune information sur le personnage et que ce dernier puisse explorer l'environnement du jeu librement rapproche Abzû de ce qu'Arsenault (2013) appelle un jeu à dominante avatorielle. Toutefois, la perspective à la troisième personne, permettant de voir les réactions du personnage-joueur et de demeurer alerte face aux éléments pouvant porter

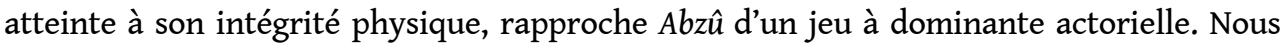
pouvons en outre remarquer la présence, dans le jeu, de marqueurs de subjectivation minimisant l'altérité entre le joueur et le personnage : le protagoniste est muet, a un 
visage peu expressif et semble découvrir son passé en même temps que le joueur. Sur le plan narratif, ces caractéristiques sont justifiées par le fait que le personnage soit un robot. Elles créent non seulement un mystère autour du personnage qui attise la curiosité du joueur, mais incitent aussi le joueur à projeter en lui sa propre personnalité et ses propres motivations.

Si l'on se fie à Therrien (2013) et Arsenault (2013), le protagoniste d'Abzû est donc un personnage idéal pour favoriser l'immersion identificatrice, parce qu'il se situe à michemin entre les approches actorielle et avatorielle, offrant à la fois un point d'ancrage dans le récit et une certaine liberté d'action. Bien entendu, le joueur peut considérer l'avatar comme l'extension de son corps et de son regard dans le jeu, tout comme il peut s'en détacher et le percevoir comme un coéquipier pour lequel il ressent une certaine empathie. Il peut également osciller entre ces deux postures et même - pour des raisons extérieures aux composantes du jeu - n'en adopter aucune. Par ailleurs, le fait que le personnage soit un robot justifie certains aspects de la jouabilité qui influencent le plan narratif, de sorte qu'il soit plus facile pour le joueur de croire au monde fictionnel et de s'y immerger. En effet, sa nature d'androïde explique non seulement que l'avatar puisse rester sous l'eau sans bonbonne d'oxygène et communiquer avec les robots-guides par le biais d'ondes sonores, mais aussi qu'il ne meurt pas après s'être fait électrocuter par des tétraèdres et qu'il acquière la force de les détruire en leur fonçant droit dessus.

\section{Influence de la dimension ludique sur l'immersion fictionnelle}

La dimension ludique n'est pas très développée dans le jeu Abzû. Les obstacles empêchant la progression sont plutôt simples à franchir : lorsqu'une porte bloque le passage d'un lieu à un autre, le personnage-joueur n'a qu'à suivre les chaînes ou les aqueducs qui y sont reliés pour trouver les manivelles qui lui permettront d'activer son ouverture. Les blessures infligées à l'avatar par les tétraèdres explosifs et les punitions réservées au joueur qui n'a pas su les éviter sont quant à elles minimes. En effet, l'électrocution du personnage-joueur a pour seule conséquence de le paralyser pendant quelques secondes et ne laisse aucune trace visible sur son corps (sauf lors de la cinématique mettant en scène la mort du requin). Le joueur comprend rapidement qu'il peut se faire électrocuter à répétition sans véritables conséquences sur la santé de son avatar ou sur sa progression dans le jeu. La multiplication d'obstacles et de punitions aurait probablement détourné l'attention du joueur de l'environnement enchanteur et de l'histoire à reconstituer ; ce qui aurait nui à son immersion sensorielle et fictionnelle. Le fait que le joueur ne soit pas contraint par une limite de temps favorise également ces deux types d'immersion, en lui permettant de s'attarder sur les paysages à couper le souffle et de scruter les fresques des temples inondés. Enfin, le but principal du jeu est intrinsèquement relié au contenu narratif, soit détruire les tétraèdres électroniques pour restaurer la vie dans l'océan.

Pour toutes ces raisons, nous aimerions avancer l'idée que, dans Abzûu, la dimension ludique n'est pas tant au service de l'immersion basée sur le défi qu'au service de l'immersion sensorielle et fictionnelle. La simplicité des défis et la clémence des punitions empêchent qu'une intense immersion basée sur le défi court-circuite la possibilité d'une immersion sensorielle et d'une immersion fictionnelle respectivement fondées sur la contemplation et la reconstitution de l'histoire. 


\section{Influence de la spatialité sur l'immersion fictionnelle}

36 Bien que l'environnement du jeu ne soit pas photoréaliste, la richesse de ses détails, ses couleurs et ses formes stimule non seulement les sens du joueur d'une manière qui favorise l'immersion sensorielle, mais lui permet aussi de bien assimiler le monde fictionnel et de mieux comprendre l'histoire du jeu. Le contraste entre, d'une part, les lieux paisibles, larges, colorés et grouillants de vie dans lesquels le personnage navigue au début du jeu et, d'autre part, les endroits menaçants, étroits, sombres, inanimés dans lesquels s'entassent les tétraèdres explosifs, aide en effet à comprendre que ces structures pyramidales épuisent l'énergie bleue permettant à la faune et la flore sous-marines d'exister. La présence de barrières «naturelles» (récifs) agissant comme des murs invisibles justifie, sur le plan narratif, l'incapacité du personnage-joueur à explorer certains endroits et renforce la crédibilité du monde fictionnel. Lorsque l'environnement navigable n'est pas délimité par des récifs et que le personnage-joueur s'aventure trop loin, l'avatar est automatiquement ramené dans la bonne direction comme s'il était repoussé par le courant. Ces éléments aident le joueur à ne pas se perdre dans l'environnement sous-marin, tout en préservant l'impression de pouvoir explorer les lieux librement. Le fait qu'il y ait peu de risques de s'égarer favorise l'immersion sensorielle et fictionnelle, dans la mesure où le joueur n'a pas à détourner son attention de la beauté des paysages ou de l'histoire pour tenter de retrouver son chemin. Nous pouvons alors avancer l'idée que, dans Abzû, la spatialité favorise non seulement l'immersion sensorielle, mais également l'immersion diégétique et narrative du joueur.

\section{Influence des contrôles sur l'immersion fictionnelle}

À première vue, la dimension kinesthésique d'Abzû semble la seule qui fait obstacle à l'immersion fictionnelle, du moins pendant la période d'adaptation du joueur. Puisque les contrôles sont sensibles et contre-intuitifs ${ }^{2}$, il est difficile pour le joueur de se sentir en phase avec le corps de son avatar et, donc, d'entrer en immersion identificatrice. Le fait que le personnage semble reprendre conscience au début du jeu vient par contre justifier, sur le plan narratif, qu'il nage maladroitement et se cogne la tête au fond de l'océan durant un certain temps. Pour empêcher que les contrôles nuisent à l'immersion sensorielle et diégétique, l'environnement spatial des premiers tableaux est toutefois large, dépourvu d'obstacle et sillonné de courants marins unidirectionnels qui facilitent le déplacement du personnage et le poussent dans la bonne direction. Le personnagejoueur peut donc progresser rapidement et contempler le paysage même s'il nage encore maladroitement. Les morceaux du puzzle narratif à reconstituer et les tétraèdres à éviter ne se présentent pas au début du jeu ; ce qui laisse suffisamment de temps au joueur pour apprendre à nager. Les dimensions spatiale, narrative et ludique s'adaptent donc aux difficultés du jeu sur le plan kinesthésique. Ceci illustre bien à quel point c'est l'équilibre entre les différentes dimensions d'un jeu qui permet de générer une expérience immersive optimale. Puisque les contrôles sensibles rendent l'évitement des tétraèdres explosifs plus difficile et augmentent les risques d'électrocution, ils finissent éventuellement par contribuer à la contagion émotionnelle et au développement d'un sentiment d'empathie à l'égard du personnage, tout en aidant le joueur à réaliser que les tétraèdres explosifs nuisent à la vie sous-marine. En ce sens, les contrôles sensibles 
favorisent à long terme l'immersion identificatoire et narrative, en plus d'être au service de l'immersion basée sur le défi.

\section{Influence de la narrativité sur l'immersion basée sur le défi}

résultat d'une interprétation libre des règles par le joueur, nous aimerions avancer l'idée qu'un jeu peut aussi naître de l'interprétation libre d'un récit lorsqu'il se présente comme un puzzle à résoudre et comme le principal défi du jeu, tel que c'est le cas pour Abzû. Dans ce type de jeu, la narrativité n'est plus seulement une accroche (hook) qui pique la curiosité du joueur et l'embarque (lures) dans le jeu (Ryan, 2006, p. 286), mais aussi un élément qui stimule l'imaginaire du joueur, captive son attention et le force à réfléchir tout au long de sa progression dans le jeu. Nous aimerions donc suggérer que, dans Abzu, plusieurs éléments narratifs nourrissent la dimension ludique du jeu et concourent eux aussi à l'immersion basée sur le défi en plus de participer à l'immersion fictionnelle. Si, à première vue, Abzû semble privilégier l'immersion fictionnelle et sensorielle, le fait que la reconstitution de l'histoire du jeu présente une énigme à résoudre permet de générer une

Sciences du jeu, 9 | 2018 
immersion basée sur le défi qui n'entre pas en concurrence avec l'immersion fictionnelle et l'immersion sensorielle.

\section{Conclusion}

41 Bref, dans $A b z \hat{u}$, les dimensions ludique, spatiale et kinesthésique ne sont pas uniquement au service de l'immersion basée sur le défi et de l'immersion sensorielle, mais également de l'immersion fictionnelle. La simplicité des défis à surmonter, la faible menace qui pèse sur le personnage, l'absence de contrainte temporelle et la linéarité de l'environnement minimisant les risques de se perdre permettent aux joueurs d'enrichir leur alterbiographie en interagissant avec les créatures sous-marines, ainsi que de concentrer leur attention sur la reconstitution de l'histoire à partir d'une série d'indices. Quant aux contrôles difficilement maîtrisables, ils ne favorisent pas l'adoption d'une posture de type « extension de soi » au début du jeu, mais finissent par encourager l'empathie réflexe en maximisant les risques d'électrocution. L'analyse du jeu Abzû permet de constater que le contenu narratif et l'avatar d'un jeu vidéo ne sont pas les seuls éléments qui ont une incidence sur les possibilités d'immersion fictionnelle du joueur. Elle démontre aussi qu'inversement, la narrativité peut concourir à l'immersion basée sur le défi lorsque l'histoire du jeu se présente comme un casse-tête à résoudre, forçant le joueur à réfléchir pour élaborer des interprétations et déchiffrer le message véhiculé par le jeu.

Cet article visait à illustrer comment les diverses dimensions d'un jeu peuvent être simultanément mises au service de plusieurs types d'immersion. Il avait également pour objectif de démontrer que les catégories de l'immersion (sensorielle, fictionnelle, basée sur le défi, diégétique, identificatrice, narrative), élaborées par les théoriciens du jeu vidéo, sont utiles à des fins d'analyse, mais ne doivent pas masquer la complexité du phénomène de l'immersion en donnant l'impression qu'elles sont mutuellement exclusives, qu'elles se font nécessairement obstacle ou encore qu'elles opèrent en vase clos.

\section{BIBLIOGRAPHIE}

AARSETH, E. (1997), Cybertext: Perspective on Ergodic Literature, Baltimore, Johns Hopkins University Press.

ALDRED J. (2014), « Characters », in M.J.P. Wolf et B. Perron (dir.), The Routledge Companion to Video Game Studies, New York, Routledge, pp. 355-363.

ARSENAULT D. (2006), Jeux et enjeux du récit vidéoludique : la narration dans le jeu vidéo, Mémoire de maîtrise, Montréal, Université de Montréal.

ARSENAULT D. (2013), « Qui est “je” ? Autour de quelques stratégies vidéoludiques de design de personnage pour gérer l'actantialité ludonarrative du joueur et son immersion fictionnelle », in R. Bourassa et L. Poissant (dir.), Avatars, personnages et acteurs virtuels, Sainte-Foy, PUQ, pp. 249-272. 
ARSENAULT D. (2014), « Narratology », in M.J.P. Wolf et B. Perron (dir.), The Routledge Companion to Video Game Studies, New York, Routledge, pp. 475 à 483.

ARSENAULT D. \& PICARD M. (2008), « Le jeu vidéo entre dépendance et plaisir immersif : les trois formes d'immersion vidéoludique ", Actes de colloque. HomoLudens. Le jeu vidéo : un phénomène social massivement pratiqué. Congrès de l'ACFAS http://ludicine.ca/sites/ludicine.ca/files/ arsenault,-picard---le-jeu-video-entre-dependance-et-plaisir-immersif_0.pdf

BONENFANT M. (2010), Sens, fonction et appropriation du jeu : l'exemple de World of Warcraft, thèse de doctorat, Montréal, Université du Québec à Montréal.

CALLEJA G. (2011), In-Game. From Immersion to Incorporation, Cambridge, The MIT Press.

CSIKSZENMIHALYI M. (1990), Flow: the psychology of optimal experience, New York, Harper \& Row.

ERMI L. \& MÄYRÄ F. (2005), « Fundamental Components of the Gameplay Experience: Analysing Immersion ", Proceedings of DiGRA 2005 Conference: Changing Views - Worlds in Play, http:// www.digra.org/wp-content/uploads/digital-library/06276.41516.pdf

FRASCA, G. (1999), « Ludology Meets Narratology. Similitude and Differences Between (Video) Games and Narrative », Parnasso, 3, pp. 365-371.

GEORGES F. (2013), «L'immersion fictionnelle dans le jeu vidéo. Le cas de Silent Hill », Nouvelle revue d'esthétique, 11, pp. 51-61.

GENVO S. (2006), « Les conditions de validité de l'immersion vidéoludique : pour une approche descriptive de la jouabilité », Actes du colloque Ludovia 2006, pp. 1-15, http://

www.ludologique.com/publis/Ludovia_Genvo_S.pdf

HENRIOT J. (1989), Sous couleur de jouer, Paris, José Corti.

JENKINS H. (2004), « Game Design as Narrative Architecture », in P. Harrington et N. FrupWaldrop (dir.), First Person, Cambridge, The MIT Press, pp. 118-130.

JUUL, J. (2001), « Game Telling Stories? A Brief Note on Games and Narratives », Game Studies, 1 (1) http://www.gamestudies.org/0101/juul-gts/

JUUL J. (2011 嘼2005區), Half-real. Video Games Between Real Rules and Fictional Worlds, Cambridge, The MIT Press.

LEVINE K. (2008), « Making them Care About Your Stupid Story ». Game Developers Conference 2008, Los Angeles.

LOMBARD M. \& DITTON T. (1997), «At the Heart of It All: The Concept of Presence ", Journal of Computer-Mediated Communication, 3 (2).

MURRAY J. (1997), Hamlet on the Holodeck: The future of narrative in cyberspace, Cambridge, The MIT Press.

RYAN M.-L. (2001), Narrative as Virtual Reality. Immersion and Interactivity in Literature and Electronic Media, Baltimore, JHU Press.

RYAN M.-L. (2006), Avatars of Story, Minneapolis, University of Minnesota Press.

RYAN M.-L. (2015 [2001]), Narrative as Virtual Reality 2. Revisiting Immersion and Interactivity in Literature and Electronic Media, Baltimore, JHU Press.

SALEN, K. \& ZIMMERMAN E. (2004), Rules of Play. Game Design Fundamentals, Cambridge, The MIT Press.

SCHAEFFER J.-M. (1999), Pourquoi la fiction ?, Paris, Seuil. 
SICART M. (2009), The Ethics of Computer Games, Cambridge, The MIT Press.

SLATER M. \& WILBUR, S. (1997), « A Framework for Immersive Virtual Environments (Five): Speculations on the Role of Presence in Virtual Environments ", Presence, 6 (6), pp. 603-616.

THERRIEN C. (2011), Illusion, idéalisation, gratification. L'immersion dans les univers de fiction à l'ère du jeu vidéo, thèse de doctorat, Université de Montréal.

THERRIEN C. (2013), « La présence vidéoludique : de l'illusion à la projection dans l'écosystème affectif de la fiction », in R. Bourassa et L. Poissant (dir.), Avatars, personnages et acteurs virtuels, Sainte-Foy, PUQ, pp. 224-247.

THERRIEN C. (2014), «Immersion », in M.J.P. Wolf et B. Perron (dir.), The Routledge Companion to Video Game Studies, New York, Routledge, pp. 451-458.

THERRIEN, C. (2017), « From Video Games to Virtual Reality (and Back) Introducing HACS (Historical-Analytical Comparative System) for the Documentation of Experiential Configuration in Gaming History ", DiGRA '17 - Proceedings of the 2017 DiGRA International Conference.

WITMER B. G. \& SINGER M. J. (1998), « Measuring Presence in Virtual Environments: A Presence Questionnaire ». Presence, 7 (3), pp. 225-240.

\section{NOTES}

1. Ainsi, le jeu de plongée Abzû joue explicitement sur le sens premier du terme " immersion ».

2. Il faut pousser le manche (thumbstick) vers le haut pour aller vers le bas et vice versa. Le joueur peut toutefois modifier les paramètres du jeu pour inverser les contrôles.

\section{RÉSUMÉS}

Cet article vise à mettre en lumière la manière dont les différentes composantes d'un jeu interagissent pour susciter l'immersion fictionnelle du joueur. En adoptant une approche ludonarrative et en s'inspirant du modèle holistique de l'immersion développé par Calleja de même que du SCI-Model d'Ermi et Mäyrä, nous chercherons à démontrer la complexité de l'immersion fictionnelle, qui a tendance à être masquée par la création de catégories et de souscatégories de l'immersion. Notre objectif est double: montrer que les différents types d'immersion ne sont pas mutuellement exclusifs et expliquer comment les éléments non narratifs d'un jeu peuvent agir sur l'immersion fictionnelle. Pour illustrer ces idées et identifier différentes façons dont les effets combinés des composantes d'un jeu peuvent influencer l'immersion fictionnelle, nous effectuerons une analyse détaillée du jeu d'aventure et d'exploration Abzû, qui invite les joueurs à s'immerger - au sens propre comme au sens figuré dans un monde sous-marin coloré et grouillant de vie.

This article is intended to show how game components interact to favor the player's fictional immersion. With the help of a ludonarrative approach and in light of Calleja's holistic model of immersion as well as Ermi and Mäyrä's SCI-Model, we intend to demonstrate the complexity of fictional immersion, which tends to be masked by the creation of categories and subcategories of 
immersion. Our objective is twofold: showing that the different types of immersion are not mutually exclusive and explaining how non-narrative game elements can influence fictional immersion. To illustrate these ideas and to identify the many ways that combined effects of game components can impact fictional immersion, we will carry out an analysis of the adventure and exploration game $A b z \hat{u}$, that is aimed to immerse the player - literally and figuratively - in a colorful underwater world teeming with marine life.

INDEX

Keywords : fictional immersion, video game, narration, character, gameplay, spatiality

Mots-clés : immersion fictionnelle, jeu vidéo, narrativité, personnage, jouabilité, spatialité

\section{AUTEURS}

\section{GABRIELLE TRÉPANIER-JOBIN}

Université du Québec à Montréal

\section{ALEXANE COUTURIER}

Université du Québec à Montréal 